\title{
Weight concerns among boys in the Republic of Ireland
}

\author{
C. Kelly ${ }^{1}$, A. Fitzgerald ${ }^{2}$, M. Molcho ${ }^{1}$ and S. Nic Gabhainn ${ }^{1}$ \\ ${ }^{1}$ Health Promotion Research Centre, National University of Ireland Galway, Ireland and ${ }^{2}$ School of Psychology, \\ University College Dublin, Ireland
}

Body weight and body image concerns are common among adolescents and various strategies are used to achieve an 'ideal' body weight or shape ${ }^{(1)}$. The type of body weight concern can differ by gender and associated risk and protective factors observed for girls may also differ for boys.

For this study, data from the 2006 Irish Health Behaviour in School-aged Children study were employed to investigate the prevalence of weight concerns among boys in Ireland. In addition, because the family environment has the potential to influence weight concerns ${ }^{(2-4)}$, associations between family meal times, relationships and family structure and reported weight concerns among boys were explored.

Health Behaviour in School-aged Children is a school-based survey with data collected through self-completion questionnaires administered in the classroom. Health Behaviour in School-aged Children Ireland collected data in 2006 from school children, aged 10-18 years ( $n$ 10334). Ethical approval was granted for the study and consent from schools, parents and children was obtained.

Boys who reported that they were currently dieting or doing something else to lose weight and those who expressed a desire to lose weight were classified as having a body weight 'loss' concern. Those boys who reported a desire to gain weight were classified as those with a body weight 'gain' concern. Associations between family meal times, communication, maternal employment, family structure and weight concerns were expressed in OR from logistic regression analysis in PASW 18.

\begin{tabular}{lccc}
\hline & $10-11$ & $12-14$ & $15-17$ \\
& years & years & years \\
& $\%(n)$ & $\%(n)$ & $\%(n)$ \\
\hline No weight concern & $74.3(413)$ & $69.3(1747)$ & $68.5(1418)$ \\
Weight 'loss' concern & $23.2(129)$ & $24.9(627)$ & $22.0(455)$ \\
Weight 'gain' concern & $2.5(14)$ & $5.8(147)$ & $9.5(197)$ \\
\hline
\end{tabular}

\begin{tabular}{|c|c|c|c|c|}
\hline & Desire to lose weight & $n$ & Desire to gain weight & $n$ \\
\hline Breakfast with mother/father every day & $0.73 * * *$ & 4748 & $0.57 * * *$ & 3904 \\
\hline Evening meal with mother/father every day & 0.93 & 4745 & $0.71 * *$ & 3899 \\
\hline Open communication with father & $0.66^{* * *}$ & 4343 & $0.50 * * *$ & 3600 \\
\hline Open communication with mother & $0.67 * * *$ & 4414 & $0.55^{* * *}$ & 3656 \\
\hline Father present in the home & 1.19 & 4498 & 1.12 & 3687 \\
\hline Mother working & 0.98 & 4537 & 1.09 & 3723 \\
\hline
\end{tabular}

$* * * P<0.001 ; * * P<0.01 ; * P<0.05$. Analyses were controlled for age.

A desire to gain weight increased with age, whereas weight 'loss' concerns remained relatively stable with age. Boys who reported frequent family meal times and good communication with their mothers and fathers were less likely to report any weight concern.

Body weight concerns among boys in Ireland are prevalent. Communicating these findings to parents and practitioners alike is important. Further exploration of both the risk and protective factors for weight concerns among boys will broaden the evidence base and have important implications for practice.

Health Behaviour in School-aged Children Ireland was funded by the Department of Health and Children, Ireland.

1. Bearman S \& Presnell K (2006) J Youth Adolesc 35, 217-229.

2. Neumark-Sztainer D, Larson NI, Fulkerson JA et al. (2010) Public Health Nutr 13, 1113-1121.

3. Pearson N, MacFarlane A, Crawford D et al. (2009) Appetite 52, 668-674.

4. Al Sabbah H, Vereecken C, Elgar F et al. (2009) BMC Public Health 9, 52. 